\title{
Spatial corrections for reactivity measurement in lead accelerator driven system
}

\author{
Pawet Gajda ${ }^{1, *}$, and Michat Orliński ${ }^{1}$ \\ ${ }^{1}$ AGH University of Science and Technology, Faculty of Energy and Fuels, Department of Nuclear \\ Energy, al. Mickiewicza 30, 30-059 Kraków, Poland
}

\begin{abstract}
One of the key areas of the development of Accelerator Driven Systems (ADS) are reactivity monitoring techniques. Since the measurement in the future industrial reactor have to be made in the real time applied methods should be accurate, simple and robust. Therefore methods based on point kinetic model are considered. Necessary experimental validation of selected methods was carried out within research project FP7 FREYA using VENUS-F reactor. This paper presents results obtained using the Sjöstrand method and the source multiplication method. Since ADS core behaviour differs from the point kinetics obtained reactivity value depends on the detector position in the system. From the results it is clear that measurement results strongly depend on the position of the detector in the system. For the Sjöstrand method these spatial effects can be successfully corrected using MCNP-calculated correction factors. Those correction factors do not change within the range of reactivity changes covered in the experiments. Spatial effects affecting source multiplication method are more complex and they depend also on neutron flux distribution in the core.
\end{abstract}

\section{Accelerator driven systems}

The Accelerator Driven System (ADS) is composed of three main components: nuclear reactor working in subcritical state, spallation neutron source and a proton accelerator. The idea of such device is relatively old and war first proposed by E. Lawrence in the early 1950's [1]. However no such industrial scale device was ever constructed. The revival of this idea is observed over last two decades, this time as one of the technologies for transmutation of spent nuclear fuel.

Growing interest in the solutions to spent nuclear fuel issue alternative to the geological storage is observed worldwide. This strategy is called partitioning and transmutation (P\&T) and has advantage of reusing large portion of the spent fuel. The three main benefits of such approach are limited overall volume of waste, limited required storage time and better fuel economy. P\&T technologies are partly used at the industrial scale today, mainly as reusing plutonium in form of the MOX fuel in regular light water reactors. However, the fully closed fuel cycle requires new technologies to increase the ability of the reactor to burn transuranic elements, especially minor actinides.

\footnotetext{
* Corresponding author: pgajda@agh.edu.pl
} 
The main advantage of the ADS is fact that the reactor is working in the subcritical state which leads to enhanced safety properties by increasing the safety margin to prompt supercriticality. It is critical when dealing with minor actinides in the fuel which lead to disadvantageous changes in such safety parameters as reactivity coefficients and delayed neutron fraction. It causes limitation of minor actinides content in the fuel for critical reactors. The enhanced safety margin in ADS can compensate for disadvantageous proprieties of minor actinides and leads to more efficient transmutation. Number of dedicated actinide burners required to close the fuel cycle can be therefore reduced using ADS in compare to use of critical fast reactors [2].

Because the reactor works in the subcritical state the reactivity of the core has to be known at any time during operation of the reactor. This requirement is a relatively new issue, which did not occur in critical reactors used to date. Appropriate reactivity monitoring methods need to be developed and tested. The key requirements for utilised method are accuracy and robustness, but also simplicity since reactivity must be determined in real-time [3].

\section{Experimental set-up}

A set of experiments devoted to validation of the reactivity monitoring techniques was performed within European Project FP7 FREYA (Fast Reactors Experiments for Hybrid Applications). Similar experiments where previously conducted in previous projects like MUSE in FP5 and EUROTRANS in FP6, however it was the first time the measurement where performed in the lead subcritical reactor, therefore in conditions close to future industrial ADS. The experiments described in this paper were performed at VENUS-F reactor at SCK-CEN facility in Mol, Belgium by international group of scientists.

The VENUS-F reactor is a zero-power research reactor. It uses metallic uranium fuel placed in the solid lead matrix. Use of lead makes it the first mock-up of fast neutron spectre lead ADS and, as was stated before allows for neutronics parameters close to the industrial ADS. The reactor core itself is a $12 \times 12$ square matrix, that can be filled with fuel assemblies or lead bars. It is then surrounded by a lead reflector. Different loading patterns are possible, with different number of fuel assemblies used, to allow for several core configurations with different neutron multiplication. During the experiments described in this paper the reactor was in $\mathrm{SC} 1$ configuration with $\mathrm{k}_{\mathrm{eff}} \approx 0.96$. The cross section of this configuration is shown in the Fig. 1. The neutron source is placed in the centre of the core surrounded by 93 fuel assemblies (marked violet in the figure). There are also six safety rods (light blue) and two control rods (red). The rest of the core matrix is filed with lead assemblies (yellow) serving as the neutron reflector. This part is called the inner reflector while the cylindrical lead structure surrounding whole core matric called the outer reflector.

The reactor is a versatile device that can work both in critical and subcritical configuration. In the latter mode four fuel assemblies are removed from the centre of the core and replaced by D-T neutron source of the GENEPI-3C neutron generator [4]. The reactor is then driven by $14 \mathrm{MeV}$ neutrons from the generator.

The GENEPI generator is able to work in three modes: pulsed, continuous and beam-trips (continuous with short, periodical interruptions of the beam). In experiments presented in this paper the generator worked in both continuous and pulsed mode (PNS).

A set of fission chambers was placed in the reactor for measuring the neutron flux. The exact positions of the fission chambers in the system are also shown in the Fig. 1. 


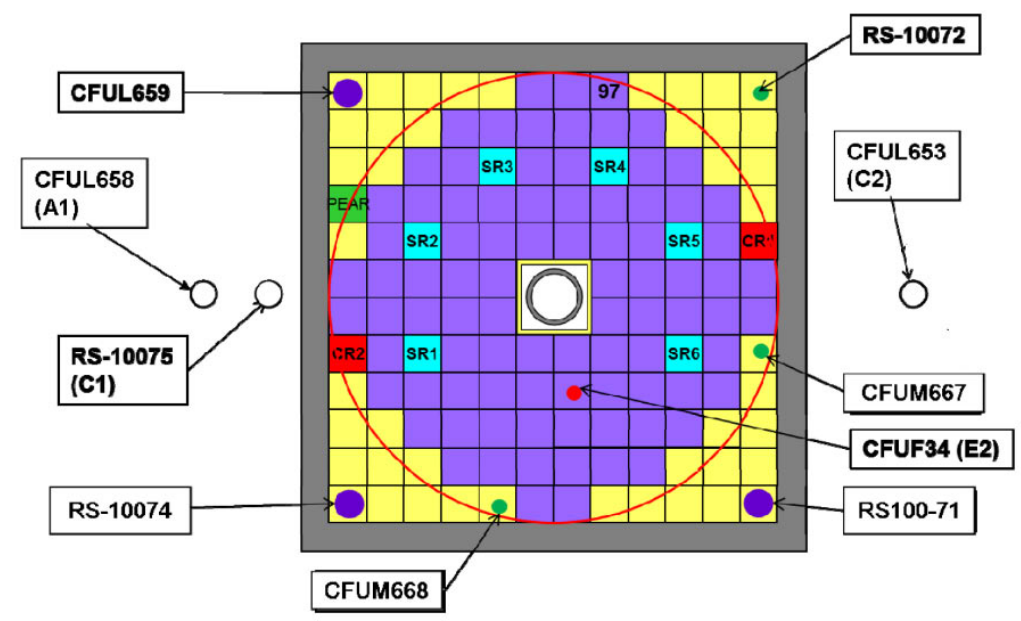

Fig. 1. VENUS-F reactor cross section and detector placement in the system [5]

\section{The Sjöstrand method}

In the first experiment described in this paper the generator was working in the pulsed mode. It then produce $600 \mathrm{~ns}$ long neutron pulses with $200 \mathrm{~Hz}$ frequency. The time evolution of the neutron flux in the system was then measured after every neutron pulse to assess the reactivity. The Sjöstrand method (sometimes also called the area method) was used to obtain the reactivity value [6].

The reference value of $\rho=5.28(13) \$$ was used for all the experiments for core configuration SC1 with both control rods in position CR@479.3 mm. It was measured using MSM method and mentioned CR position corresponds to criticality point achieved in the critical core configuration [7,8]. For other CR positions the reference values were obtained using MCNP simulation code to calculate reactivity difference to the reference point. MCNPX 2.5 with JEFF 3.1 data libraries was used for all calculations presented in this paper.

\subsection{Preliminary results}

Reactivity values for SC1 core with control rods in different positions calculated basing on neutron flux measured by every detector are shown in the Table 1. Graphic comparison of the results and reference value for selected case (CR@479.3 mm) is shown in the Fig. 2.

It is clearly visible that obtained reactivity value depends on detector placement in the reactor. Only detector placed in the active part of the core (CFUF34) is showing reactivity values consistent with the reference. The other detectors are giving values to criticality. This overestimation grows with distance from the core centre. This so called spatial effects are caused by the fact that behaviour of the system varies from the point kinetic model on which the method is based. Also the neutron flux spatial distribution in subcritical systems is more uneven than in critical reactors. The flux distribution is also changing over time after the neutron pulse from the source. This phenomena is then affecting the reactivity assessment with the Sjöstrand method. Similar effects were observed before in other subcritical systems, such as YALINA [9]. It should be noted that in case of detectors placed in the outer reflector the prompt neutron flux decay is not fully observed which can also affect the measurement. 
Table 1. Reactivity values obtained with the Sjöstrand method

\begin{tabular}{|c|c|c|c|c|}
\hline \multirow{2}{*}{ Detector } & \multicolumn{4}{|c|}{$\boldsymbol{\rho}[\mathbf{\$}]$} \\
\cline { 2 - 5 } & CR@600mm & CR@479.3mm & CR@240mm & CR@0mm \\
\hline CFUF34 & $-5.09(8)$ & $-5.25(9)$ & $-5.69(18)$ & $-6.30(13)$ \\
\hline CFUM667 & $-4.85(3)$ & $-4.99(3)$ & $-5.79(11)$ & $-6.02(7)$ \\
\hline CFUM668 & $-4.87(3)$ & $-4.98(3)$ & $-5.64(10)$ & $-5.98(7)$ \\
\hline RS-10071 & $-4.81(1)$ & $-5.00(1)$ & $-5.55(1)$ & $-5.96(2)$ \\
\hline RS-10072 & $-4.81(1)$ & $-4.99(2)$ & $-5.61(3)$ & $-5.95(2)$ \\
\hline RS-10074 & $-4.84(1)$ & $-5.03(1)$ & $-5.62(3)$ & $-5.98(2)$ \\
\hline CFUL659 & $-4.75(1)$ & $-4.92(1)$ & $-5.50(1)$ & $-5.88(1)$ \\
\hline RS-10075 & $-4.78(2)$ & $-4.90(3)$ & $-5.44(3)$ & $-5.81(4)$ \\
\hline CFUL658 & $-4.48(3)$ & $-4.61(3)$ & $-5.10(4)$ & $-5.43(4)$ \\
\hline CFUL653 & $-4.62(2)$ & $-4.78(3)$ & $-5.26(3)$ & $-5.62(3)$ \\
\hline
\end{tabular}

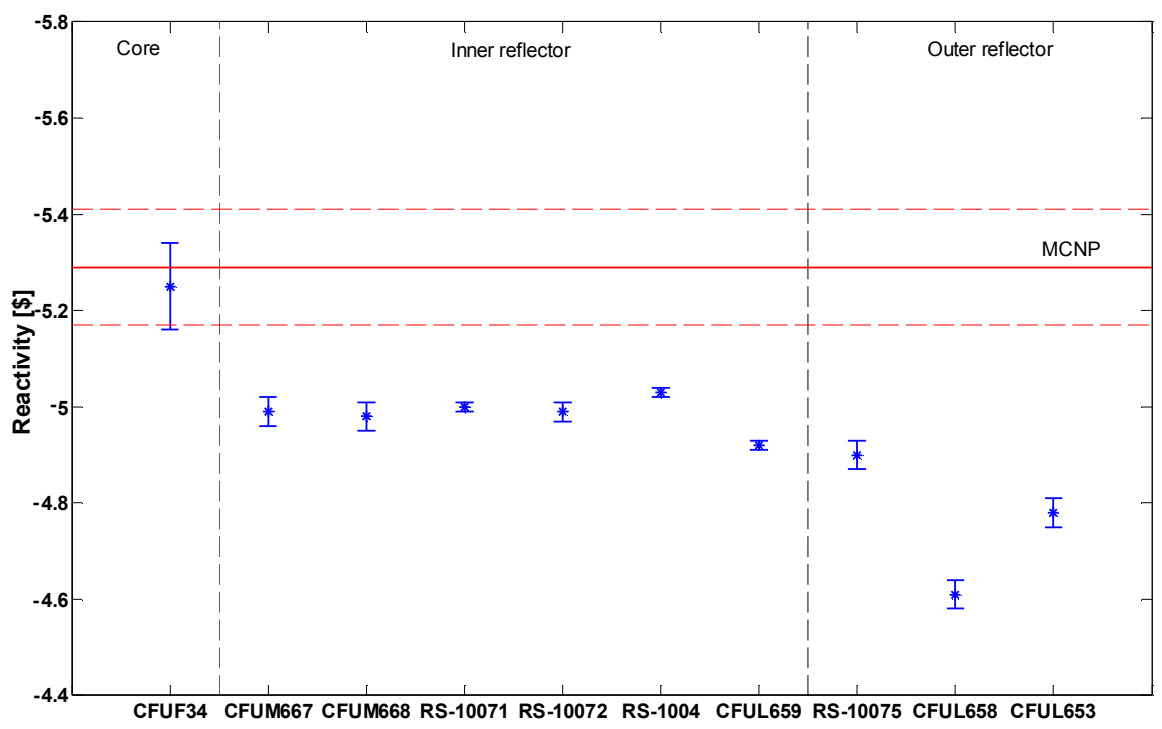

Fig. 2. Results for the Sjöstrand method - CR@479.3 mm

\subsection{Spatial effects correction}

For the method to be proved suitable for ADS there is a need to correct observed spatial effects. The correction method applied in this case is based on calculating the correction factors for each detector position with the MCNP simulation code [10]. The whole procedure requires calculating the neutron flux for each every detector with and without taking into account delayed neutron as well as core reactivity. It is then possible to calculate the expected reactivity value given by the detector placed in that position. Since the same relation between core reactivity and expected result from the detector should occur both in simulation and experiments, they calculated relations of those values can be then used to correct the experimental values. 
The correction factors were calculated separately for different control rod positions used in the experiments. However their values do not significantly change with core reactivity and once calculated correction factor can be used at least in same range of reactivity.

The results after applying the corrections are shown in Table 2 and Fig. 3. Corrected results show good agreement both with reference values and between different detectors. Only exception are the detectors placed in the outer reflector, where despite applying the corrections obtained values are still higher than those obtained in the rest of the system. However, as it was stated before, the full prompt neutron flux decay wasn't observed in this detectors. This phenomena can fully explain the discrepancy of those results even after applying the spatial correction. Using lower frequency of the pulses to allow for full prompt decay should allow to obtain proper result. For all other detectors proposed correction method was proved suitable to use in this subcritical system. Similar results were achieved by other groups involved in the FREYA projects [11, 12].

Table 2. Spatially corrected reactivity values obtained with the Sjöstrand method

\begin{tabular}{|c|c|c|c|c|}
\hline \multirow{2}{*}{ Detector } & \multicolumn{4}{|c|}{$\boldsymbol{\rho}[\mathbf{~}$} \\
\cline { 2 - 5 } & CR@600mm & CR@479.3mm & CR@240mm & CR@0mm \\
\hline CFUF34 & $-5.08(9)$ & $-5.23(10)$ & $-5.70(19)$ & $-6.34(14)$ \\
\hline CFUM667 & $-5.12(5)$ & $-5.25(5)$ & $-6.12(13)$ & $-6.33(9)$ \\
\hline CFUM668 & $-5.17(5)$ & $-5.25(5)$ & $-5.97(12)$ & $-6.36(9)$ \\
\hline RS-10071 & $-5.13(5)$ & $-5.31(5)$ & $-5.91(5)$ & $-6.30(6)$ \\
\hline RS-10072 & $-5.14(5)$ & $-5.30(5)$ & $-5.99(6)$ & $-6.33(6)$ \\
\hline RS-10074 & $-5.09(4)$ & $-5.31(5)$ & $-5.90(6)$ & $-6.32(6)$ \\
\hline CFUL659 & $-5.02(4)$ & $-5.19(5)$ & $-5.83(5)$ & $-6.26(5)$ \\
\hline RS-10075 & $-5.04(5)$ & $-5.15(5)$ & $-5.74(6)$ & $-6.13(7)$ \\
\hline CFUL658 & $-4.78(5)$ & $-4.90(5)$ & $-5.45(6)$ & $-5.78(7)$ \\
\hline CFUL653 & $-4.94(5)$ & $-5.09(5)$ & $-5.63(6)$ & $-5.99(6)$ \\
\hline
\end{tabular}

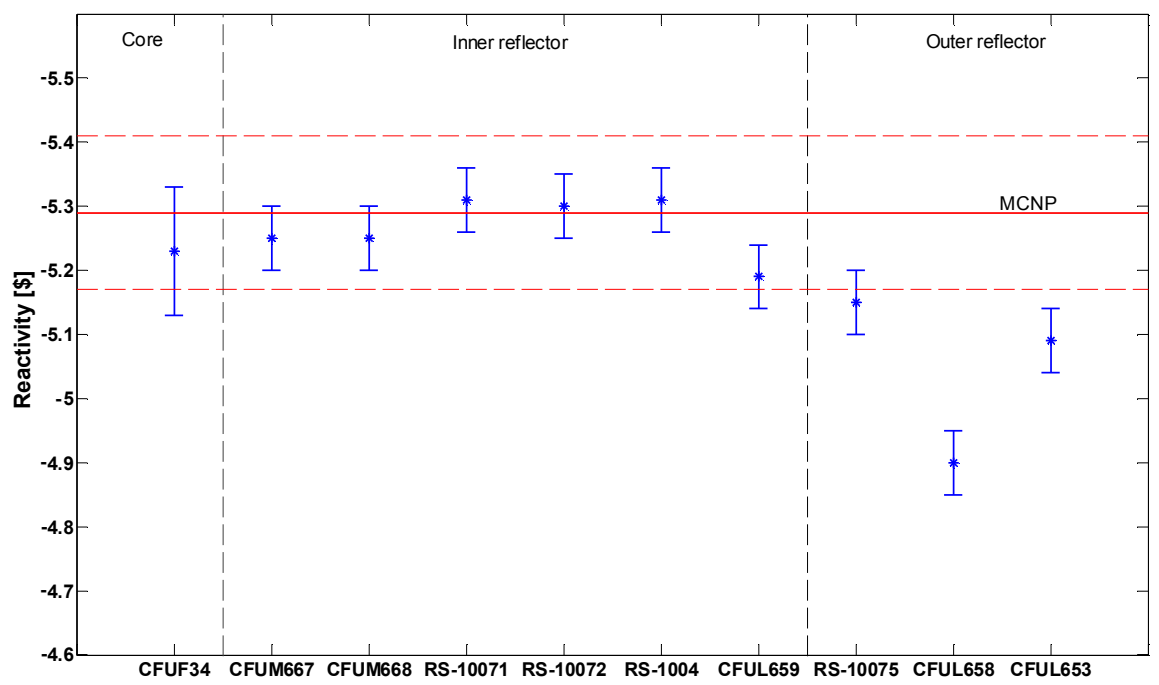

Fig. 3. Results for the Sjöstrand method after corrections - CR@479.3 mm 


\section{The source multiplication method}

In this set of experiments the generator was operated in the continuous mode and the control rods here moved step by step between extreme positions separately or together. The source multiplication method was used to determine core reactivity [13]. In this method the change in the neutron multiplication factor is described by following formula:

$$
\frac{C R_{2}}{C R_{1}}=\frac{1-k_{e f f 1}}{1-k_{e f f 2}}
$$

where $C R_{l}$ is neutron count rate in the detector before change of the reactivity, $C R_{2}$ is the count rate after the change and $k_{\text {eff } I}$ and $k_{\text {eff } 2}$ are neutron multiplication factors before and after the change of reactivity.

It should be noted that this method allows for measuring only relative changes in the reactivity. All values where therefore calculated in relation to the reference value for control rods position CR@479.3 mm mentioned before.

\subsection{Measurement results}

The results are presented in the Table 3. They are shown as measured reactivity change caused by control rods movement as measured by each detector.

It is clearly visible that once again the obtained results depends on the detector position. However in this case the relation between detector placement and measured reactivity changes is different. Because the measurement is based on static neutron flux measurement instead of its evolution in time the key factor affecting measured reactivity changes are local flux disturbances caused by the control rods which can be greater that the global change of the neutron flux in the core. This effect is visualised in the Fig. 4.

Table 3. Reactivity change due to CR insertion measured with the source multiplication method

\begin{tabular}{|c|c|c|c|}
\hline \multirow{2}{*}{ Detector } & \multicolumn{3}{|c|}{$\Delta \mathbf{k}$ [pcm] } \\
\cline { 2 - 4 } & $\begin{array}{c}\text { CR1 \& CR2 } \\
0-600 \mathrm{~mm}\end{array}$ & $\begin{array}{c}\text { CR1 0-600mm } \\
\text { CR2@479.3mm }\end{array}$ & $\begin{array}{c}\text { CR2 0-600mm } \\
\text { CR1@479.3mm }\end{array}$ \\
\hline CFUF34 & $763(36)$ & $371(21)$ & $401(23)$ \\
\hline CFUM667 & $941(41)$ & $542(28)$ & $416(23)$ \\
\hline CFUM668 & $838(38)$ & $463(25)$ & $586(30)$ \\
\hline RS-10071 & $846(39)$ & $611(31)$ & $420(24)$ \\
\hline RS-10072 & $913(40)$ & $606(31)$ & $422(24)$ \\
\hline RS-10074 & $911(40)$ & $404(23)$ & $603(32)$ \\
\hline CFUL659 & $848(38)$ & $406(23)$ & $598(31)$ \\
\hline RS-10075 & $1238(61)$ & $413(23)$ & $641(33)$ \\
\hline CFUL658 & $1296(63)$ & $421(24)$ & $670(34)$ \\
\hline CFUL653 & $1217(61)$ & $638(32)$ & $428(24)$ \\
\hline $\begin{array}{c}\text { MCNP } \\
\text { (simulation) }\end{array}$ & $841(16)$ & $408(16)$ & $418(16)$ \\
\hline
\end{tabular}

It is clearly visible that measured reactivity change is bigger in detectors placed in proximity of the control rod used. What is important, unlike in PNS experiment and Sjöstrand method, the measured reactivity change is dependent not only on detector position but also on which control rod was used to cause the change. It is possible to correct observed 
discrepancies by modified source multiplication method which relies on correction factors to compensate such local changes of the neutron flux [14]. Those have to be calculated not only for each detector but also for each case of control rod movement separately. It would therefore require significant additional calculation effort. However preliminary assessment of the reactivity is possible without using the correction factors by choosing the detectors placed as far away as possible from the moving control rod [15].

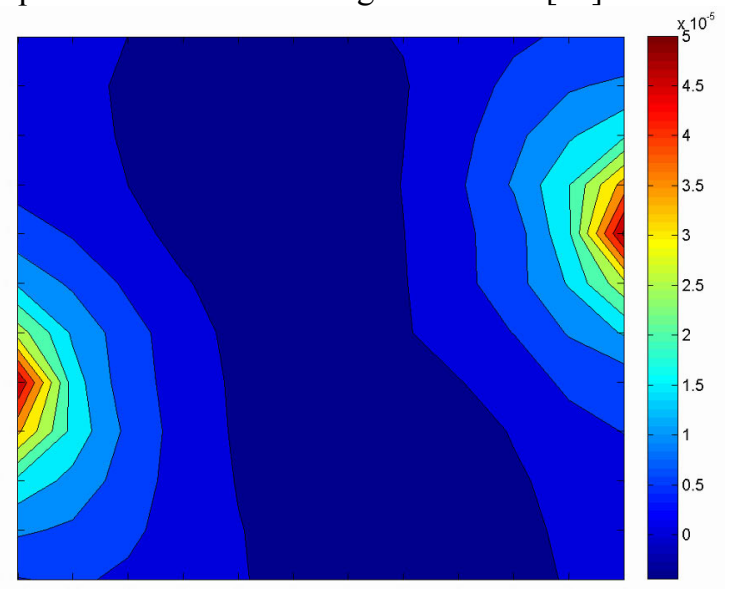

Fig. 4. Neutron flux change with both control rods fully extracted and inserted

Despite fact that uneven rod insertion wasn't used in PNS experiments described before the correction factors were calculated for such case to assess if such uneven flux distribution would affect therm. The results are shown in the Table 4. For the whole range of control rods positions that can be achieved in this core configuration the correction factors are not affected neither by change of reactivity, nor by asymmetrical rod insertion.

Table 4. Correction factors for the Sjöstrand method in the core with uneven CR insertion

\begin{tabular}{|c|c|c|c|c|}
\hline \multirow{2}{*}{ Detector } & \multicolumn{4}{|c|}{ Correction factor } \\
\cline { 2 - 5 } & $\begin{array}{c}\text { CR1@600mm } \\
\text { CR2@600mm }\end{array}$ & $\begin{array}{c}\text { CR1@600mm } \\
\text { CR2@0mm }\end{array}$ & $\begin{array}{c}\text { CR1@0mm } \\
\text { CR2@600mm }\end{array}$ & $\begin{array}{c}\text { CR1@0mm } \\
\text { CR2@0mm }\end{array}$ \\
\hline CFUF34 & $1.001(8)$ & $0.998(8)$ & $1.000(8)$ & $0.994(7)$ \\
\hline CFUM667 & $0.948(7)$ & $0.948(7)$ & $0.949(7)$ & $0.951(8)$ \\
\hline CFUM668 & $0.942(7)$ & $0.945(7)$ & $0.943(9)$ & $0.94(7)$ \\
\hline RS-10071 & $0.937(7)$ & $0.943(7)$ & $0.941(8)$ & $0.946(7)$ \\
\hline RS-10072 & $0.936(8)$ & $0.941(8)$ & $0.938(9)$ & $0.940(8)$ \\
\hline RS-10074 & $0.951(7)$ & $0.946(9)$ & $0.948(7)$ & $0.946(8)$ \\
\hline CFUL659 & $0.946(8)$ & $0.943(9)$ & $0.941(8)$ & $0.940(9)$ \\
\hline RS-10075 & $0.949(9)$ & $0.951(9)$ & $0.948(9)$ & $0.948(8)$ \\
\hline CFUL658 & $0.938(9)$ & $0.937(11)$ & $0.941(9)$ & $0.939(9)$ \\
\hline CFUL653 & $0.936(10)$ & $0.938(10)$ & $0.935(10)$ & $0.939(10)$ \\
\hline
\end{tabular}

\section{Summary and conclusions}

Methods used to determine the reactivity are derived from point kinetic model and are affected by so called spatial effects, meaning that obtained value depends on detector position 
within the system. Such result is consistent with previous experiments and was confirmed for fast spectre subcritical system, in conditions more similar to possible future industrial ADS than in previous experiments. It was shown that in PNS measurement the spatial effects can be corrected by calculating proper correction factors for every detector position. However, use of this method in real-time measurement would require calculating the correction factors in advance. The next step should be therefore an assessment of range of conditions that once calculated correction factor can be used and its dependence on reactivity, neutron flux distribution, etc. In the set of conditions covered by the experiments the correction factors did not change, however it has covered a reactivity range of less than $1.5 \$$.

Spatial effects affecting source multiplication method are more complex and therefore more difficult to correct. Because it can measure only relative changes of reactivity, in authors view it should be used only for preliminary assessment supplementary to other, more accurate method.

We appreciate the efforts and support of all the scientists and institutions involved in the FREYA project. Financial support by the European Commission (through the contracts \#269665) and by the Polish Ministry of Science and Higher Education is gratefully acknowledged (contract no 11.11.210.200).

\section{References}

1. J.L. Heilbron, R.W. Seidel, B.R. Wheaton, Lawrence and His Laboratory - A historian's view of the Lawrence Year, Chapter 5: Cold War in Science (1996). Retrieved from http://www.lbl.gov/Science-Articles/Research-Review/Magazine/1981/

2. Accelerator-driven Systems (ADS) and Fast Reactors (FR) in Advanced Nuclear Fuel Cycles: A Comparative Study (OECD Publications, Paris, 2002)

3. P. Baeten, H. Ait Abderrahim, Progress in Nuclear Energy, 43, 413-419 (2003)

4. A. Billebaud, P. Baeten, H. Ait Abderrahim, et al., The GUINEVERE Project for Accelerator Driven System Physics, International Conference GLOBAL 2009 The Nuclear Fuel Cycle: Sustainable Options \& Industrial Perspectives (Paris, 2009)

5. A. Billebaud, FREYA SC1 detector configuration, FP7 FREYA internal document (LPCS Grenoble, 2012)

6. N.G. Sjöstrand, Arkiv för fysik, 11, 13 (1956)

7. J.L. Lecouey et al., Recommended value for reactivity (SC1) and related business, FREYA WP1 Meeting 30-31 Oct 2014, Grenoble (2014)

8. J.L. Lecouey, N. Marie, G. Ban, et al., Ann. of Nucl. Energy, 83, 65-75 (2015)

9. P. Gajda, J. Janczyszyn, W. Pohorecki, Nukleonika: Intern. J. Nucl. Res., 58(2), 287$293(2013)$

10. V. Bécares, E. Gonzales, D. Villamarin, et al., Ann. of Nucl. Energy, 53, 331-341 (2013)

11. N. Marie (LPCC), G. Lehaut (LPCC), J.L. Lecouey, et al., Reactivity monitoring using the area method for the subcritical VENUS-F core within the framework of the FREYA Project, arXiv:1306.1063 [physics.ins-det] (2013)

12. W. Uyttenhove, D. Lathouwers, J.-L. Kloosterman, et al., Ann. of Nucl. Energy, 72, 286-297 (2014)

13. M. Tsuji, N. Suzuki, Y. Shimazu, J. of Nuc. Sc. and Tech., 40(3), 158-169 (2003)

14. T. Endo, A. Yamamoto, Y. Yamane, Ann. of Nucl. Energy, 38(11), 2417-2427 (2011)

15. J. Cetnar, G. Domańska, P. Gajda, J. Janczyszyn, Nukleonika: Intern. J. Nucl. Res., 59(4), 137-143 (2014) 